\title{
Research Paper: Erythropoietin Pretreatment Effect on Blood Glucose and Its Relationship With Inflammatory Factors After Brain Ischemic-Reperfusion Injury in Rats
}

\author{
Raheleh Gholamzadeh $^{1}$, Mehdi Eskandari ${ }^{1}$, Mohammad Reza Bigdeli $^{2,3} \odot$ Hossein $^{\text {Mostafavi }}{ }^{{ }^{*}} \odot$
}

1. Department of Physiology and Pharmacology, School of Medicine, Zanjan University of Medical Sciences, Zanjan, Iran.

2. Department of Physiology, Faculty of Life Sciences and Biotechnology, Shahid Beheshti University, Tehran, Iran.

3. Institute for Cognitive and Brain Sciences, Shahid Beheshti University, Tehran, Iran.

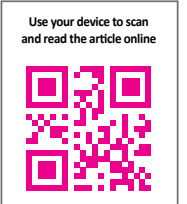

Citation: Gholamzadeh, R., Eskandari, M., Bigdeli, M. R., \& Mostafavi, H. (2018). Erythropoietin Pretreatment Effect on

Blood Glucose and Its Relationship With Inflammatory Factors After Brain Ischemic-Reperfusion Injury in Rats. Basic and Clinical Neuroscience, 9(5), 347-356. http://dx.doi.org/10.32598/bcn.9.5.347

\section{(C) $0(3)$}

Funding: See Page 353

Article info:

Received: 31 August 2017

First Revision: 23 September 2017 Accepted: 30 April 2018

Available Online: 01 September 2018

Keywords:

Erythropoietin, Pretreatment, Blood glucose, Inflammatory factors, Brain ischemia

\section{A B S T RA C T}

Introduction: Brain Ichemic-Reperfusion Injury (IRI) activates different pathophysiological processes. It also changes physiological parameters such as Blood Glucose (BG) level. An increase in BG after stroke is associated with poor clinical outcomes. Erythropoietin has been shown to be effective on both reducing inflammation and BG level. Therefore, in this study the erythropoietin pretreatment effect on BG and its relationship with inflammatory markers after brain IRI was investigated.

Methods: Thirty adult male Wistar rats were randomly divided into 5 groups: sham, control and 3 pretreatment groups: single dose, double dose, and triple dose that received $1000 \mathrm{U} / \mathrm{kg}$ of erythropoietin before stroke induction in different times intraperitoneally. A rat model of IRI was established by Middle Cerebral Artery Occlusion (MCAO) for 60 minutes. Infarct volume, neurological defects, Interleukin- $1 \alpha$ (IL-1 $\alpha$ ) and IL-6 serum levels were evaluated 24 hours after reperfusion. Also BG was measured after 1, 6, and 24 hours.

Results: Single dose of erythropoietin significantly decreased infarct volume and improved neurological defects which was associated with decreased serum level of IL- $1 \alpha$ and IL- 6 but higher doses of erythropoietin administration had adverse effects on histological, neurological, and inflammatory results. In addition, erythropoietin significantly increased BG in a dosedependent manner.

Conclusion: Erythropoietin could reduce brain IRI by reducing inflammation and BG stabilization. The results of the present study demonstrated a relationship between inflammatory factors and hyperglycemia after IRI and suggested that erythropoietin may be useful for preventing brain IRI, but its higher doses should be used with caution due to possible side effects.

\footnotetext{
* Corresponding Author:

Hossein Mostafavi, PhD

Address: Department of Physiology and Pharmacology, School of Medicine, Zanjan University of Medical Sciences, Zanjan, Iran.

Tel: +98 (24) 33440286

E-mail: mostafavi73@gmail.com
} 


\section{Highlights}

- Brain ischemia increases blood glucose, inflammatory factors, and neurological deficits.

- There is a relationship between increment of blood glucose, inflammation, and clinical outcomes.

- Erythropoietin pretreatment improves outcome after brain ischemia by decreasing inflammation and blood glucose stabilization.

- Administration of erythropoietin in high dose has side effects and should be used with caution.

\section{Plain Language Summary}

Stroke is one of the most important causes of mortality in the world. Many conditions, for example heart bypass operation, transplantation, sickle cell anemia, and so on are risk factors for stroke. After the stroke, the physiological functions of the body, such as blood pressure, blood sugar, body temperature, cell metabolism, oxygen saturation, etc., are impaired and in this situation different pathological process are activated. These physiological changes have important effects on increasing stroke damage, which are often neglected in experimental studies. Attention to association of physiological and pathological agents have clinical importance in the prevention of stroke. Therefore, in this study, we examined the erythropoietin pretreatment effect on blood glucose, inflammatory factors and its association with stroke volume and clinical outcomes. The results of our study showed that administration of erythropoietin protects the brain against stroke, but this drug should be used with caution in high-risk individuals because of its side effects and possibility of stroke volume increase.

\section{Introduction}

I

schemic stroke is the leading cause of death in the worldwide that imposes high costs on individuals and society (Yuen et al., 2011). Heart bypass surgery, transplantation, sickle cell anemia and heart attack are risk factors for cerebral ischemia (Bigdeli \& Mohagheghi, 2014). Despite its enormous advances, medical science still lacks a safe and effective treatment for stroke (Yip et al., 2011). Thrombolytic therapy is the only safe and effective method for acute management of ischemic stroke which has a golden time and usage limitation (Yuen et al., 2011). Recently, preconditioning mechanisms, like pharmacological precondi-tioning are used to increase tolerance to ischemia (Gholamzadeh, Eskandari, Mostafavi, \& Bigdeli, 2016).

Cerebral ischemia leads to the activation of inflammatory processes, and different metabolic and electrophysiological disorders (Bigdeli \& Mohagheghi, 2014; Shah, 2000). Reperfusion after ischemic injury is associated with a greater harm, i.e. Ischemic-Reperfusion Injury (IRI) (Bigdeli \& Mohagheghi, 2014; Paschos, Lykissas, \& Beris, 2008; Wu et al., 2014). In addition, cerebral ischemia changes body physiological conditions such as blood oxygen saturation, blood sugar, temperature and blood pressure (Radermecker \& Scheen, 2010). Hyper- glycemia is common in the early stroke phase and has been observed in two-thirds of all types of ischemic stroke (Lindsberg \& Roine, 2004). Studies demonstrated that hyperglycemia is associated with worse outcomes, enhanced infarct volume and increased risk for poststroke mortality (Bhalla, Wolfe, \& Rudd, 2001; Lindsberg \& Roine, 2004; Mehta, 2003). Oxidative glucose metabolism is perturbed following ischemia and causes lactic acidosis and vasogenic edema (Bhalla et al., 2001).

Hyperglycemia increases the production of lactic acid, leading to neuronal damage (Bhalla et al., 2001; Mehta, 2003). Also, both hyperglycemia and hypoglycemia lead to an increased production of free radi-cals and inflammatory factors such as Interleukin-1 (IL-1) and IL-6 (Shukla, Shakya, Perez-Pinzon, \& Dave, 2017). A neuroendocrine stress and inflammatory response may contribute to increased blood glucose after a stroke. Thus, maintaining physiological homeostasis and reducing inflammation after a stroke has an important role in therapeutic achievements and mortality reduction (Wong \& Read, 2008).

Erythropoietin is a glycoprotein hormone. It is secreted by the liver in fetal and synthesized in the kidney in adults (Chen et al., 2015; Wu et al., 2014). Erythropoietin was initially applied to treat anemia (Yao et al., 2016). The protective effect of erythropoietin preconditioning on reducing IRI and inflammatory responses has been 
investigated in the brain (Yu, Fan, Sun, Yao, \& Chai, 2016), heart (Rong \& Xijun, 2015), kidney (Elshiekh, Kadkhodaee, Seifi, Ranjbaran, \& Ahghari, 2015; Liao, Li, Wang, \& Xie, 2016), intestine (Kai-Lan \& Si, 2015), liver (Liu et al., 2015), and lung (Wu et al., 2006). Erythropoietin could reduce infarct size and improve neurological deficits (Yuen et al., 2011).

Several studies have determined the effects of erythropoietin on decreased blood glucose and an increased glucose tolerance in diabetic rats (Chen et al., 2015; Meng, Zhu, Bi, Yang, \& Wang, 2013; Niu, Chang, Niu, Cheng, \& Lee, 2016). To our knowledge, no study has explored the effects of erythropoietin pretreatment on blood glucose in non-diabetic stroke patients and its relationship with inflammatory factors and outcomes. Therefore the present study investigated the impact of erythropoietin on improved IRI by reducing blood glucose and inflamma-tory factors.

\section{Methods}

\subsection{Experimental groups}

According to Figure 1, 30 adult male Wistar rats (weighing 200 to $300 \mathrm{~g}$ ) were maintained under standard conditions (12:12 h light-dark cycle and controlled temperature of $24 \pm 2^{\circ} \mathrm{C}$ ). Then, the rats were ran-domly divided into 5 groups (6 rat in each group): sham (receiving surgical stress), control (Middle Cerebral Artery Occlusion [MCAO] model receiving saline 0.9\%) and 3 pretreatment groups: single dose pre-treatment group
(30 minutes before the stroke induction), double dose ( 0.5 and 48 hours before), triple dose $(0.5,48$ and 96 hours before the stroke induction) that received 1000 $\mathrm{U} / \mathrm{kg}$ of recombinant human erythropoietin alpha (ampoule Eprex 4000 unit, code: 1228048326 prepared by Pooyesh Darou Pharmaceuticals company) intraperitoneally before the stroke induction (Figure 1).

The animals were anesthetized 30 minutes after the last injection by chloral hydrate (Merck, Germany, Code: 102425). Brain ischemia was induced by MCAO model for 60 minutes. Then, the animals were evaluated in terms of stroke volume, blood glucose, inflammatory factors (IL1- $\alpha$ \& IL6) and motor neurological deficits 24 hours after the stroke induction.

\subsection{Induction of focal cerebral ischemia by MCAO model}

To close the Middle Cerebral Artery (MCA) and induction of ischemia, the weighed animals were anesthetized with chloral hydrate $(400 \mathrm{mg} / \mathrm{kg})$ and placed in the supine position on the surgical pad. Under car-diac monitoring, MCAO surgery model was performed in accordance with Longa et al. method. The surgical site was sterilized with $70 \%$ alcohol. A linear $2-\mathrm{cm}$ incision was made on the right side of the neck along the spine of the animal. Under a microscopic surgery by pushing the salivary tissue and sternohyoid muscle, common carotid artery appeared which was separated from the vagus nerve. Then, a silicone coated size 3-0 inch nylon suture with a round tip was entered to the external carotid artery and

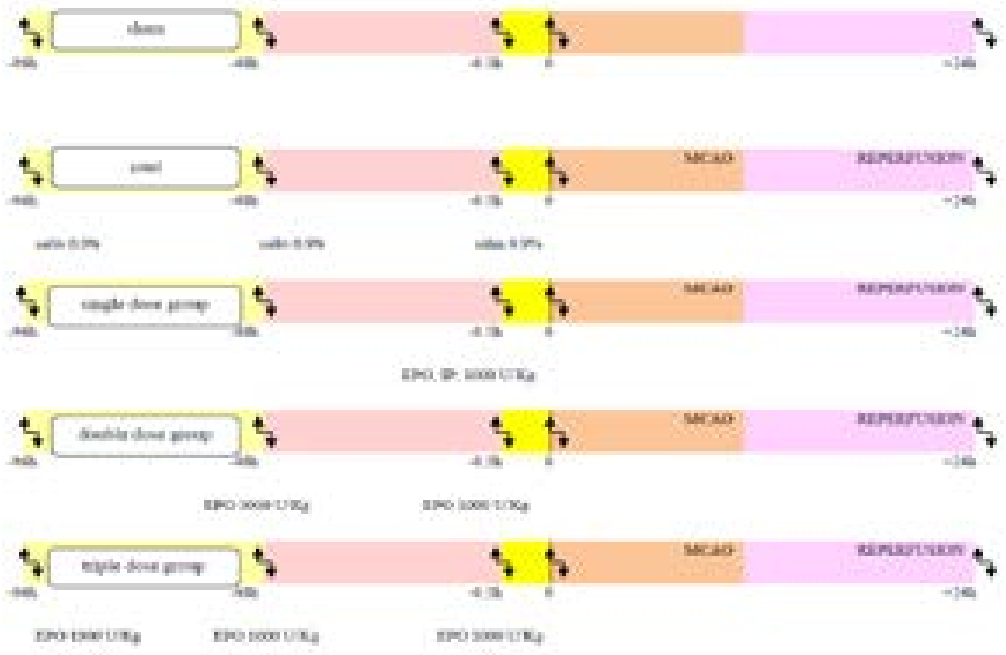

Figure 1. Schematic representation of the experimental groups

Stroke induced by middle cerebral artery occlusion model and blood flow was established after 1 hour. Tissue and blood parameters were assessed 24 hours after the reperfusion. 

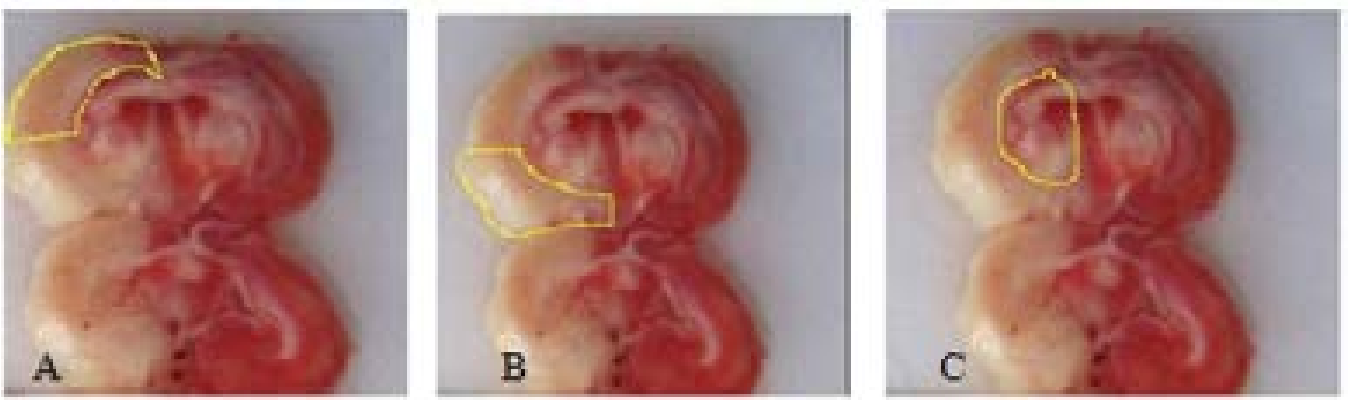

Figure 2. Separation of penumbra (A), core (B) and subcortex (c) by Image-J Software

pushed through internal carotid artery to reach the MCA. A slight resistance against suture after passing 20-22 mm of suture length indicated that it is placed in the correct location. Blood flow was restored after 60 minutes. During the surgery, rectal body temperature was measured and maintained at about $37^{\circ} \mathrm{C}$ (Bigdeli, 2008; Chiang, Messing, \& Chou, 2011).

\subsection{Measurement of stroke volume}

The animals were sacrificed by chloral hydrate $(800 \mathrm{mg} /$ $\mathrm{kg}$ ) and decapitated 24 hours after induction of ischemia, to measure infarct volume. Then, their brain was removed immediately and placed in cool nor-mal saline $\left(4^{\circ} \mathrm{C}\right)$ for 10 minutes. Seven brain coronal sections were cut at 2 $\mathrm{mm}$ in thickness from frontal to temporal lobes, according to rat brain matrix. Tissue sections were immersed in triphenyltetrazolium chloride (Sigma, USA, code: 1.38380) solution and incubated at $37^{\circ} \mathrm{C}$ for 10 minutes. Then, images were taken from sections using a digital camera and transferred to a computer for analysis by Image-J Software. As shown in Figure 2, stroke volume was calculated according to the following formula in core, penumbra, and subcortex regions (Bigdeli, 2008; Yuen et al., 2011).

Brain infarct volume $=$

Left hemisphere volume - ( Right hemisphere volume - Infarct volume) Left hemisphere volume

\subsection{Measurement of blood glucose}

Blood glucose levels were controlled at 1,6 and 24 hours after the stroke induction by a glucometer (Accu-Chek Performa ) using the animals' tail vein blood samples (Chen et al., 2015).

\subsection{Inflammatory factors of interleukin-1a and in- terleukin-6}

Two milliliters of the rats' blood samples were taken to measure IL- $1 \alpha$ and IL-6, 24 hours after the induction of stroke. Blood samples were transferred to sterile tubes and centrifuged at $4000 \mathrm{rpm}$, at $4^{\circ} \mathrm{C}$. The serum samples were stored at $-70^{\circ} \mathrm{C}$ in microtubes. Serum levels of IL- $1 \alpha$ and IL-6 were measured by ELISA method according to Diaclone France protocol kit (Gul, Yasim, \& Aral, 2009).

\subsection{Assessment of motor neurological deficits}

Animals' neurological defects were evaluated by standard Bederson criteria. The animals' neurological status were scored 24 hours after the induction of ischemia. The interpretation of scores is as follows:

Score (0): No neurological deficits were observed; Score (1): Complete failure on the anterior surface of the front paws (mild focal neurological deficits); Score (2): Turning to the left (moderate focal neurologi-cal deficits); Score (3): Falling to the left (severe focal neurological deficits); Score (4): Animal is incapable of walking spontaneously and has a low level of consciousness; and Score (5): Death (Bigdeli, 2008).

\subsection{Statistical analysis}

The collected results were analyzed by SPSS using one-way Analysis of Variances (ANOVA) and LSD posthoc analysis. Also, neurological deficits were analyzed by Mann-Whitney $U$ test. The obtained re-sults are expressed as Mean \pm SEM and $\mathrm{P}<0.05$ is considered as statistically significant.

\section{Results}

\subsection{Stroke volume and motor neurological deficits}

A single dose of erythropoietin pretreatment significantly reduced total stroke volume and stroke volume of core and penumbra regions. Also it improved neurological deficit scores compared to the control group $(\mathrm{P}<0.05)$. Double and triple doses of erythropoietin pretreatment had no effect on stroke volume and neurological deficit scores. Moreover, significant differences were observed between pretreatment groups with regard to infarct volume and neurological deficits $(\mathrm{P}<0.05)$. (Figure 3 and Table 1 ) 
(A)

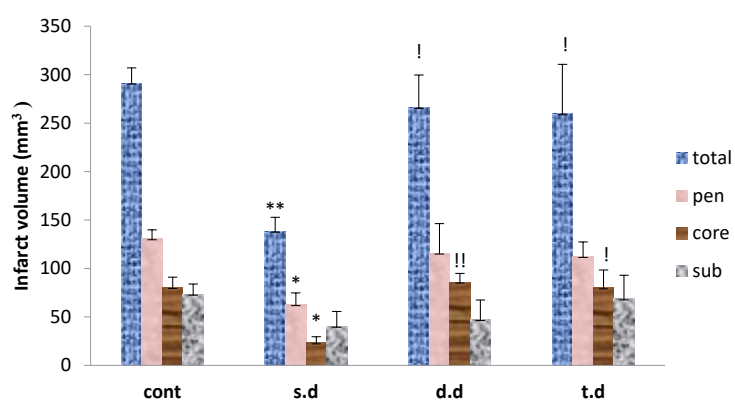

(B)

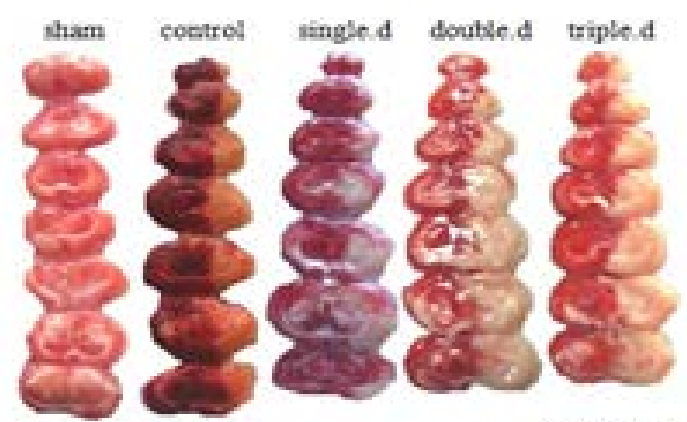

NEUR JSCIENCE

Figure 3. Comparison of erythropoietin pretreatment effect $(1000 \mathrm{U} / \mathrm{kg})$ on tissue damage volume in the treatment groups

A: Single dose (s.d); Double dose (d.d); Triple dose (t.d) with control group in penumbra, core, subcortex and total volume.

${ }^{*} \mathrm{P}<0.05$ and ${ }^{* *} \mathrm{P}<0.01$ are significant differences compared to the controls.

$\mathrm{B}$ : Sections of brain tissue in different groups were stained with the characteristics of red in safe areas and white in the affected areas. $\mathrm{P}<0.05$ are " $\mathrm{P}<0.007$ are significant differences with single dose group.

\subsection{Blood glucose}

The blood glucose was measured at 1, 6 and 24 hours after brain ischemia in all groups which it had no significant difference in control group compared to the sham. Erythropoietin pretreatment increased blood glucose after stroke. This enhancement was significant in the double and triple dose erythropoietin pretreatment groups, compared to control and single dose groups; especially in the first and sixth hours after the stroke $(\mathrm{P}<0.05)$. Also, all experimental groups showed a reduction in blood glucose within 24 hours after the stroke (Figure 4).

\subsection{Inflammatory factors: Interleukin-1a and inter- leukin-6}

Serum inflammatory factors interleukin-1- $\alpha$ and interleukin-6 increased after stroke in all groups, compared to the sham group in which this rise was significant in IL-1 $\alpha$. A single dose of erythropoietin pretreat-ment re- duced serum levels of IL-1 $\alpha$ and IL-6, compared to the control group. However, this reduction was not significant. Double and triple dose erythropoietin pretreatment had no effect on IL-1 $\alpha$ and IL-6 serum levels among the groups (Figures 5 and 6).

\section{Discussion}

Blood flow maintenance in the ischemic area is among the main objectives in stroke treatment. However, restoration of blood flow in the damaged area may aggravate the injury. In addition, post-stroke changes in different physiological conditions of the body such as blood glucose, blood saturation, and temperature could increase the severity of the lesion. Therefore, it is important to prevent future damage after reperfu-sion. Stabilizing physiological conditions and reducing levels of inflammation can decrease the injury caused by the stroke

Table 1. Comparing neurological deficits between the control and experimental groups according to Bederson criteria

\begin{tabular}{|c|c|c|c|c|c|c|c|c|c|c|}
\hline \multirow{2}{*}{ Groups } & \multirow{2}{*}{ Median } & \multirow{2}{*}{ Mean } & \multicolumn{6}{|c|}{ Neurological Deficits Grading } & \multirow{2}{*}{ Total } & \multirow{2}{*}{ Sig. } \\
\hline & & & 0 & 1 & 2 & 3 & 4 & 5 & & \\
\hline Control & 4 & 4.066 & 0 & 0 & 2 & 3 & 2 & 8 & 15 & \\
\hline Single dose & 3 & 2.866 & 1 & 1 & 5 & 2 & 3 & 3 & 15 & $\mathrm{P}<0.05^{*}$ \\
\hline Double dose & 4 & 4.266 & 0 & 0 & 2 & 0 & 5 & 8 & 15 & $P<0.05^{\#}$ \\
\hline Triple dose & 4 & 3.666 & 0 & 1 & 3 & 2 & 3 & 6 & 15 & \\
\hline
\end{tabular}

${ }^{*} \mathrm{P}<0.05$ is the significant differences with the control group and ${ }^{\#} \mathrm{P}<0.05$ is the significant differences with double dose group. 


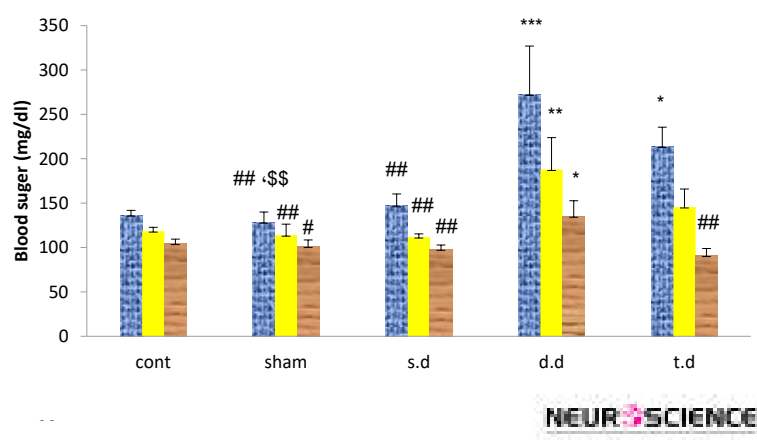

Figure 4. Blood glucose at 1, 6 and 24 hours after stroke induction in the sham, control and experimental groups

${ }^{*} \mathrm{P}<0.05,{ }^{* *} \mathrm{P}=0.003,{ }^{* * *} \mathrm{P}<0.000$ : significant differences with the controls; \# $\mathrm{P}<0.05$ : significant differences with the double dose group; ${ }^{\# \$ \$} \mathrm{P}<0.01$ : significant differences with the double dose (\#) and triple dose (\$) groups.

and reperfusion (Gentile, Seftchick, Huynh, Kruus, \& Gaughan, 2006; Wong \& Read, 2008; Yu et al., 2016).

A study demonstrated that erythropoietin pretreatment has a positive effect on the infarct volume and neurological deficits (Yu et al., 2016). The results of our study suggested that injecting a single dose of erythropoietin pretreatment $(1000 \mathrm{U} / \mathrm{kg}) 30$ minutes before the stroke induction, significantly reduced infarct volume damage and improved neurological deficits. These results are consistent with results of previous studies. However, the results regarding higher doses of erythropoietin were inconsistent with the previous experimental studies. These diverse results may be due to different time and dosage of erythropoietin admin-istration.

We recognized that pretreatment with a single dose of erythropoietin can also positively affect IRI. In contrast with the prior research, administrating a single dose of erythropoietin has better effects on IRI (which could be due to its fewer side effects) than administration of multiple doses.

In another study we reported that erythropoietin pretreatment for 48 and 96 hours were associated with a significant increase in hemoglobin, hematocrit and brain edema that may explain the adverse conse-quences due to the side effects (Bigdeli, Mostafavi, Gholamzadeh, \& Eskandari, 2016). In addition, a moderate hyperglycemia was observed in $40 \%$ of individuals without diabetes in the acute phase of stroke (Quinn, Dawson, \& Walters, 2011). Hyperglycemic and normoglycemic patients show more severe neurological defects and poor clinical outcomes (Bhalla et al., 2001; Dietrich, Alonso, \& Busto, 1993; Rader-mecker \& Scheen, 2010).

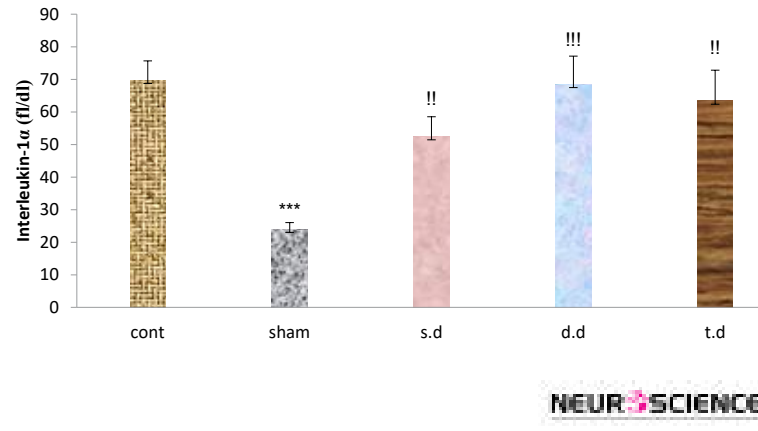

Figure 5. Serum levels of interleukin-1a 24 hours after stroke induction in the sham, control, and experimental groups

${ }^{* * *} \mathrm{P}<0.000$ indicates significant differences, compared to the controls; " $\mathrm{P}<0.01$ and "' $\mathrm{P}<0.000$ indicate significant differences, compared to the sham group.

Our research demonstrated that erythropoietin increased blood glucose levels in all groups, especially in the acute phase of stroke. The increase in blood glucose was approximately dose-dependent and higher blood glucose values were correlated with poor clinical and histological outcomes. However unlike the other studies in diabetic rats, erythropoietin increased blood sugar. Several studies have reported a possible link between erythropoietin level and hypoglycemia (Chen et al., 2015).

Hypoglycemia exacerbates stress responses. Stress response causes a further increase in blood glucose in hypoglycemia, compared to normoglycemia condition (Ceriello et al., 2012). This may suggest that erythropoietin pretreatment decreases blood glucose in non-diabetic rats before stroke induction and exacerbates hyperglycemia resulting from stress response after IRI. The lack of weight gain among the treated animals with higher doses of erythropoietin confirms this hypothesis.

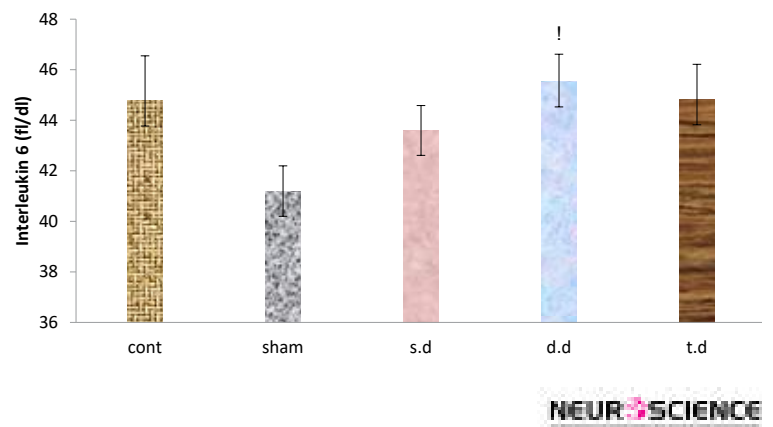

Figure 6. Serum levels of interleukin-6 24 hours after stroke induction in the sham, control and experimental groups

P $<0.05$ indicates significant differences compared to the sham group. 
Decreased glucose levels following the treatment with erythropoietin in rats with a high-fat diet for 2 weeks was associated with weight loss in them (Meng et al., 2013). Also, decreasing blood glucose over a period of 24 hours in all groups can be explained by stress response. In addition, previous studies reported a connection between blood glucose levels, inflammatory markers and the activation of stress response after stroke (Meng et al., 2013; Shukla et al., 2017). Additionally, several studies have demonstrated that ischemia-reperfusion is associated with enhanced inflammatory factors such as IL-1, IL- $1 \beta$, TNF- $\alpha$ (tumor necrosis factor-alpha), and IL-6. It is also correlated with worse histological outcomes in which erythropoietin pretreatment protected tissue from IRI by reducing these factors (Liao et al., 2016; Liu et al., 2015; Nandra et al., 2013; Rong \& Xijun, 2015; Wu et al., 2006).

In connection with inflammatory markers, we discovered that a single dose of erythropoietin pretreatment decreased levels of IL- $1 \alpha$ and IL-6, com-pared to the control group. On the other hand, the groups with higher levels of inflammatory markers showed worse tissue results. Moreover, our results identified an association between inflammatory markers and blood glucose level. Meng et al. (2013) study on high-fat diet-fed mice along with the increment of blood glucose, reported an increased in IL- 6 and TNF- $\alpha$. Based on previous studies, the main determinants of blood glucose concentration were cortisol, glucagon and insulin (O’neill, Davies, Fullerton, \& Bennett, 1991).

Ramirez reported that the treatment of anemia in dialysis patients with recombinant human erythropoietin hormone could affect the pituitary-hypothalamic-adrenal axis and increase responses of adrenocorticotropin hormone to corticotropin-releasing hormone. However, to confirm the hypothesis of hyperglycemia after hypoglycemia and stress response occurrence in brain ischemia following erythropoietin pretreatment, it is necessary to measure blood glucose before stroke induction and cortisol level in the early hours after the stroke. Evaluating the above-mentioned parameters is recommended for further studies (Ramirez, Bittle, Sanders, Rabb, \& Bercu, 1994).

\section{Conclusion}

In conclusion, our results suggest an association between hyperglycemia, levels of inflammatory factors and severity of injury after the IRI by MCAO model in rats. In addition, erythropoietin pretreatment de-creased IRI by reducing inflammatory factors, establishing stable physiological conditions, as well as improving infarct volume and neurological deficits. It appears that its effects are dose-dependent. Our results recommend that the administration of higher erythropoietin doses should be taken with caution due to its possible side effects. However, further investigations are required to determine more detailed effects of erythropoietin on physiological conditions and stress response over activation after IRI in the brain.

\section{Ethical Considerations}

\section{Compliance with ethical guidelines}

All animal experiments have been conducted in accordance with the International Standard for Animal Ethics, under the supervision of the Ethics Committee of Zanjan University of Medical Sciences.

\section{Funding}

This research did not receive any specific grant from funding agencies in the public, commercial, or not-forprofit sectors.

\section{Authors contributions}

The Authors contributions is as follows: All the affairs related to conceptualization, methodology, validation, investigation, resources, data curation and visualization of this article has been done with collaboration of all authors. Writing-original draft preparation: Raheleh Gholamzadeh; Writing-review \& editing: Raheleh Gholamzadeh; Supervision: Hossein Mostafavi; Formal \& analysis software: Mohammad Reza Bigdeli, Raheleh; and Project administration, funding acquisition: Mehdi Eskandari.

\section{Conflict of interest}

The authors certify that they have no affiliation with or involvement in any organization or entity with any financial interest, or non- financial interest in the subject matter or materials discussed in this manuscript.

\section{Acknowledgements}

All authors would like to appreciate Dr Abdolreza Esmaeil Zadeh from Immunology Department of Zanjan University of Medical Sciences and Ms. Sepideh Khaksari from Shahid Beheshti University, for their scientific cooperation.

\section{References}

Bhalla, A., Wolfe, C., \& Rudd, A. (2001). Management of acute physiological parameters after stroke. An International Journal of Medicine, 94(3), 167-72. [DOI:10.1093/qjmed/94.3.167] [PMID] 
Bigdeli, M. R. (2008). [The threshold assessment of ischemic tolerance induced by normobaric hyperoxia in rat stroke model (Persian)]. Research in Medicine, 32(2), 95-103.

Bigdeli, M., Mostafavi, H., Gholamzadeh, R., \& Eskandari, M (2016). [Pretreatment effect of erythropoietin on brain tissuewater content after brain ischemia induction by Middle Cerebral Artery Occlusion (MCAO) in male wistar rats (Persian)] International Journal of Medical Reviewes, 3(1), 389-400.

Bigdeli, M. R., \& Mohagheghi, F. (2014). The pathophysiology of brain ischemia and ischemic preconditioning. Zahedan Journal of Research in Medical Sciences, 16(2), 1-5.

Ceriello, A., Novials, A., Ortega, E., La Sala, L., Pujadas, G., Testa, R., et al. (2012). Evidence that hyperglycemia after recovery from hypoglycemia worsens endothelial function and increases oxidative stress and inflammation in healthy control subjects and subjects with type 1 diabetes. Diabetes, 61(11), 2993-7. [DOI:10.2337/db12-0224] [PMID] [PMCID]

Chen, L. N., Sun, Q., Liu, S. Q., Hu, H., Lv, J., Ji, W. J., et al. (2015). Erythropoietin improves glucose metabolism and pancreatic $\beta$-cell damage in experimental diabetic rats. Molecular Medicine Reports, 12(4), 5391-8. [DOI:10.3892/mmr.2015.4006]

Chiang, T., Messing, R. O., \& Chou, W. H. (2011). Mouse model of middle cerebral artery occlusion. Journal of Visualized Experiments, 48, e2761. [DOI:10.3791/2761]

Dietrich, W. D., Alonso, O., \& Busto, R. (1993). Moderate hyperglycemia worsens acute blood-brain barrier injury after forebrain ischemia in rats. Stroke, 24(1), 111-6. [DOI:10.1161/01. STR.24.1.111] [PMID]

Elshiekh, M., Kadkhodaee, M., Seifi, B., Ranjbaran, M., \& Ahghari, P. (2015). Ameliorative effect of recombinant human erythropoietin and ischemic preconditioning on renal ischemia reperfusion injury in rats. Nephro-Urology Monthly, 7(6), e31152. [DOI:10.5812/numonthly.31152] [PMID] [PMCID]

Gentile, N. T., Seftchick, M. W., Huynh, T., Kruus, L. K., \& Gaughan, J. (2006). Decreased mortality by normalizing blood glucose after acute ischemic stroke. Academic Emergency Medicine, 13(2), 174-80. [DOI:10.1197/j.aem.2005.08.009] [PMID]

Gholamzadeh, R., Eskandari, M., Mostafavi, H., \& Bigdeli, M. R. (2016). A review on the pretreatment effect of EPO on ischemic tolerance in different tissues with an approach to the tissue protection mechanisms. International Journal of Medical Reviews, 3(1), 389-400.

Gul, M., Yasim, A., \& Aral, M. (2009). The levels of cytokines in rats following the use of prophylactic agents in vascular graft infection. Bratislavske Lekarske Listy, 111(6), 316-20.

Kai-Lan, W., \& Si, Z. (2015). Pretreatment with erythropoietin attenuates intestinal ischemia reperfusion injury by further promoting PI3K/Akt signaling activation. Transplantation Proceedings, 47(6), 1639-45. [DOI:10.1016/j.transproceed.2015.02.023] [PMID]

Liao, J. G., Li, M. Y., Wang, X. H., \& Xie, Q. (2016). The protective effect of erythropoietin pretreatment on ischemic acute renal failure in rats. Journal of Acute Disease, 5(5), 408-12. [DOI:10.1016/j.joad.2016.08.008]

Lindsberg, P. J., \& Roine, R. O. (2004). Hyperglycemia in acute stroke. Stroke, 35(2), 363-4. [DOI:10.1161/01. STR.0000115297.92132.84] [PMID]
Liu, Q. S., Cheng, Z. W., Xiong, J. G., Cheng, S., He, X. F., \& Li, X. C. (2015). Erythropoietin pretreatment exerts anti-inflammatory effects in hepatic ischemia/reperfusion-injured rats via suppression of the TLR2/NF-KB pathway. Transplantation Proceedings, 47(2), 283-9. [DOI:10.1016/j.transproceed.2014.10.045] [PMID]

Mehta, S. (2003). The glucose paradox of cerebral ischaemia. Journal of Postgraduate Medicine, 49(4), 299-301. [PMID]

Meng, R., Zhu, D., Bi, Y., Yang, D., \& Wang, Y. (2013). Erythropoietin inhibits gluconeogenesis and inflammation in the liver and improves glucose intolerance in high-fat diet-fed mice. PloS One, 8(1), e53557. [DOI:10.1371/journal.pone.0053557] [PMID] [PMCID]

Nandra, K. K., Collino, M., Rogazzo, M., Fantozzi, R., Patel, N S., \& Thiemermann, C. (2013). Pharmacological preconditioning with erythropoietin attenuates the organ injury and dysfunction induced in a rat model of hemorrhagic shock. Disease Models \& Mechanisms, 6(3), 701-9. [DOI:10.1242/dmm.011353] [PMID] [PMCID]

Niu, H. S., Chang, C. H., Niu, C. S., Cheng, J. T., \& Lee, K. S. (2016). Erythropoietin ameliorates hyperglycemia in type 1-like diabetic rats. Drug Design, Development And Therapy, 10, 1877-84. [PMID] [PMCID]

O'neill, P., Davies, I., Fullerton, K., \& Bennett, D. (1991). Stress hormone and blood glucose response following acute stroke in the elderly. Stroke, 22(7), 842-7. [DOI:10.1161/01. STR.22.7.842] [PMID]

Paschos, N., Lykissas, M. G., \& Beris, A. E. (2008). The role of erythropoietin as an inhibitor of tissue ischemia. International Journal of Biological Sciences, 4(3), 161-8. [DOI:10.7150/ ijbs.4.161] [PMID] [PMCID]

Quinn, T., Dawson, J., \& Walters, M. (2011). Sugar and stroke: Cerebrovascular disease and blood glucose control. Cardiovascular Therapeutics, 29(6), e31-e42. [DOI:10.1111/j.17555922.2010.00166.x] [PMID]

Radermecker, R. P., \& Scheen, A. J. (2010). Management of blood glucose in patients with stroke. Diabetes $\mathcal{E}$ Metabolism, 36(3), S94-S9. [DOI:10.1016/S1262-3636(10)70474-2]

Ramirez, G., Bittle, P. A., Sanders, H., Rabb, H., \& Bercu, B. B. (1994). The effects of corticotropin and growth hormone releasing hormones on their respective secretory axes in chronic hemodialysis patients before and after correction of anemia with recombinant human erythropoietin. The Journal of Clinical Endocrinology \& Metabolism, 78(1), 63-9. [DOI:10.1210/ jcem.78.1.8288716]

Rong, R., \& Xijun, X. (2015). Erythropoietin pretreatment suppresses inflammation by activating the PI3K/Akt signaling pathway in myocardial ischemia-reperfusion injury. Experimental and Therapeutic Medicine, 10(2), 413-8. [DOI:10.3892/ etm.2015.2534] [PMID] [PMCID]

Shah, S. (2000). Stroke pathophysiology. Foundation for Education and Research in Neurological Emergencies. Retrieved from: https://pdfs.semanticscholar.org/545d/7e83d7938031a1e8179e f76757ccd34ba13c.pdf

Shukla, V., Shakya, A. K., Perez-Pinzon, M. A., \& Dave, K. R. (2017). Cerebral ischemic damage in diabetes: An inflammatory perspective. Journal of Neuroinflammation, 14(1), 21. [DOI:10.1186/s12974-016-0774-5] [PMID] [PMCID] 
Wong, A. A., \& Read, S. J. (2008). Early changes in physiological variables after stroke. Annals of Indian Academy of Neurology, 11(4), 207-20. [DOI:10.4103/0972-2327.44555] [PMID] [PMCID]

Wu, H., Ren, B., Zhu, J., Dong, G., Xu, B., Wang, C., et al (2006). Pretreatment with recombined human erythropoietin attenuates ischemia-reperfusion-induced lung injury in rats. European Journal of Cardio-Thoracic Surgery, 29(6), 902-7. [DOI:10.1016/j.ejcts.2006.02.036] [PMID]

Wu, S. K., Yang, M. T., Kang, K. H., Liou, H. C., Lu, D. H., Fu, et al. (2014). Targeted delivery of erythropoietin by transcranial focused ultrasound for neuroprotection against ischemia/ reperfusion-induced neuronal injury: A long-term and shortterm study. PloS One, 9(2), e90107. [DOI:10.1371/journal. pone.0090107] [PMID] [PMCID]

Yao, M., Ni, J., Zhou, L., Peng, B., Zhu, Y., \& Cui, L. (2016). Elevated fasting blood glucose is predictive of poor outcome in non-diabetic stroke patients: A sub-group analysis of SMART. PloS One, 11(8), e0160674. [DOI:10.1371/journal. pone.0160674] [PMID] [PMCID]

Yip, H. K., Tsai, T. H., Lin, H. S., Chen, S. F., Sun, C. K., Leu, S., et al. (2011). Effect of erythropoietin on level of circulating endothelial progenitor cells and outcome in patients after acute ischemic stroke. Critical Care, 15(1), R40. [DOI:10.1186/ cc10002] [PMID] [PMCID]

Yu, D., Fan, Y., Sun, X., Yao, L., \& Chai, W. (2016). Effects of erythropoietin preconditioning on rat cerebral ischemiareperfusion injury and the GLT1/GLAST pathway. Experimental and Therapeutic Medicine, 11(2), 513-8. [DOI:10.3892/ etm.2015.2919] [PMID] [PMCID]

Yuen, C. M., Sun, C. K., Lin, Y. C., Chang, L. T., Kao, Y. H., Yen, C. H., et al. (2011). Combination of cyclosporine and erythropoietin improves brain infarct size and neurological function in rats after ischemic stroke. Journal of Translational Medicine, 9(1), 141-55. [DOI:10.1186/1479-5876-9-141] [PMID] [PMCID] 
